\title{
Simulation study on an acceleration control system for semi-active in-car crib with joint application of regular and inverted pendulum mechanisms
}

\author{
Takeshi KAWASHIMA* \\ *Department of Mechanical Engineering, Kanagawa Institute of Technology \\ 1030 Shimo-ogino, Atsugi, Kanagawa 243-0292, Japan \\ E-mail: kawashima@eng.kanagawa-it.ac.jp
}

Received: 14 January 2017; Revised: 12 March 2017; Accepted: 16 April 2017

\begin{abstract}
To reduce the collision shock and risk of injury to an infant in an in-car crib (or a child safety bed) during a vehicle crash, it is necessary to limit the force acting on the crib below a certain allowable value. To realize this objective, we propose a semi-active in-car crib system with the joint application of regular and inverted pendulum mechanisms. The crib is supported by an arm similar to a pendulum, and the pendulum system itself is supported by an arm similar to an inverted pendulum. In addition, the arm acting as a regular pendulum is joined with the arm acting as an inverted pendulum through a linking mechanism, and the friction torque of the joint connecting the base and the latter arm is controlled using a brake mechanism, which enables the proposed in-car crib to gradually increase the deceleration of the crib and maintain it at around the target value. This system not only reduces the impulsive force but also transfers the force to the infant's back using a spin control system, i.e., the impulse force is made to act perpendicularly on the crib. The spin control system was developed in our previous work. The present work focuses on the acceleration control system. A semi-active control law with acceleration feedback is introduced using a dynamic equation for the jerking of the crib. In addition, the effectiveness of the system is demonstrated using numerical software for a multibody dynamics simulation, and some of results are reported.
\end{abstract}

Key words: Shock control, Occupant crash protection, Passive safety device, Child restraint system, Impact relaxation

\section{Introduction}

This study focuses on equipment used to ensure the safety of an infant in a car. To reduce the collision shock and injury risk to an infant in an in-car crib (or a child safety bed) during a car crash, it is necessary to limit the force acting on the crib to below a certain allowable value. That is to say, the impact force affecting the infant within a short period of time is changed to a smaller force that affects the infant for a longer period of time by moving the crib forward. However, the cabin space of a vehicle is limited. Therefore, the force must be maintained at a constant level. To realize this objective, we propose a semi-active in-car crib with a joint application of regular and inverted pendulum mechanisms, as shown in Fig. 1. The crib is supported by arm 2, which acts like a pendulum, and the pendulum system itself is supported by arm 1, which acts like an inverted pendulum. Arm 2 rotates at a difference of 30 degrees with arm 1 using a simple linking mechanism. In addition, the friction torque of the joint connecting the base and arm 1 is controlled using a braking mechanism. Therefore, the proposed in-car crib is able to gradually increase the deceleration of the crib and maintain it at around the target value. In addition, our proposed system is able to save energy using a semi-active control system, which is a significant advantage for a vehicle with a limited amount of power.

In this project, an in-car crib (or child safety bed), which is a bed-type child-seat, is applied because the risk of brain damage (encephalopathy) from a decrease in arterial oxygen saturation can be reduced. In particular, abdominal compression can be avoided, which is not the case when a child car seat is used. In addition, it can also be used for 
neonatal infants.

However, with an in-car crib, a collision impact is directed toward the infant's side, and the resulting motion of the body is relatively complex. For this reason, the use of a spin control system was proposed for the crib, allowing the impact to fall on the infant's back, i.e., the force acts perpendicularly on the crib. This control technique was developed as an actively controlled regular pendulum-type bed for use in ambulances (Kawashima, 2002). The present study therefore focuses on the development of a crib movement system.

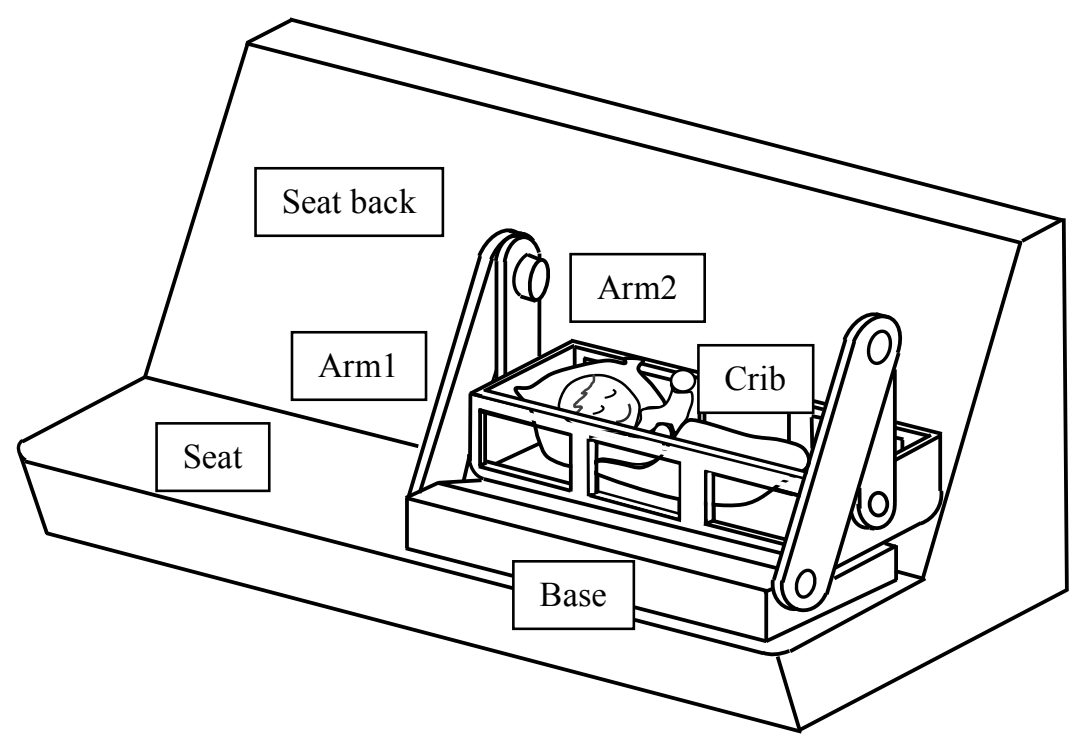

Fig. 1 Conceptual diagram of the in-car crib with a joint application of regular and inverted pendulum mechanisms to reduce the collision shock and injury risk to an infant lying in the crib during a car crash.

Many different pendulum mechanisms have been proposed for crib movement systems. In a patented design by Sawaishi (2004), a child car seat is described as a rotating seat, similar to a pendulum, which is aimed at reducing the impact on a baby pressed against the seatbelt and redirecting the force toward the seat. When a child car seat is supported in this manner, the initial deceleration acting on the seat can be reduced almost completely by moving the seat. However, this deceleration cannot be reduced further after the pendulum has rotated (Kawashima, 2012 and 2014). Therefore, regular pendulum-type in-car cribs are unsuitable when large impulsive forces are involved. In our proposed in-car crib, the deceleration of the crib can be maintained at almost a constant level during a collision, which is one of the main advantages of the proposed system. A child car seat that is rotated using electromagnets installed on the seat and base to reduce the impact forces and redirect the force toward the child's hip has been registered as a utility model (Tamura, 2000). In addition, a patent for a child car seat that is rotated to a safe position before a collision using predictive information of a vehicle crash to reduce harm to the baby was developed (Ono et al., 2008). With these two systems, the child car seat is moved by an actuator using power during or before a collision. In our proposed system, the rotation of the arms supporting the crib is controlled semi-actively using a braking mechanism, resulting in a reduction in the impulsive force. That is, our proposed system saves energy, which is a significant advantage for a vehicle with a limited amount of power, and is another major advantage.

An inverted pendulum-type active in-car crib was previously proposed to reduce the impulsive force acting on the crib to an allowable level during crib movement during a car crash (Kawashima, 2010). This crib is supported by an arm, which also acts like an inverted pendulum. In a vehicle cabin, the space for the crib movement is limited. To minimize the impulsive force in such a restricted space, the force acting on the crib must be maintained at a constant level, from the initial stage until the final collision stage. The arm is initially tilted backward because of the difficulty of movement of the inverted arm. Therefore, the deceleration of the crib can be maintained at less than the vehicle deceleration until the arm reaches an upright position. In addition, a semi-active shock control system is applied to maintain the deceleration at a constant level. Although this system is effective during a car crash with strong impulsive forces, it is not effective during a car crash with weak forces because the crib does not move (Kawashima, 2012 and 2014). 
To combine the advantages of a regular pendulum-type in-car crib and an inverted pendulum-type in-car crib, we propose an in-car crib that involves the joint application of both regular and inverted pendulum mechanisms. In this system, the deceleration of the crib increases gradually, and is maintained at below the vehicle deceleration, thereby resulting in a system with advantages of both a regular pendulum mechanism and an inverted pendulum mechanism (Kawashima, 2012 and 2014).

We also propose a semi-active control system. First, we developed a control algorithm that adjusts only the damping coefficient of the joint connecting the base and the arm acting as an inverted pendulum. We confirmed the effectiveness of the system using numerical simulations. The results indicate that the deceleration of the crib increases gradually and is maintained at around the target value of $26 \mathrm{G}$ when the deceleration of the base fixed on the vehicle seat is $30 \mathrm{G}$ (Kawashima, 2012). Subsequently, we developed a control algorithm that adjusts the friction torque of the joint connecting the base and the arm acting as an inverted pendulum. We also confirmed the effectiveness of the modified system through numerical simulations. The results indicate that the deceleration of the crib increases gradually and is maintained at around the target value of $25 \mathrm{G}$ when the deceleration of the base fixed on the vehicle seat is $30 \mathrm{G}$ (Kawashima, 2014). We then developed a control algorithm that adjusts the friction torque of the joint connecting the base and the arm acting as an inverted pendulum, as well as the joint connecting this arm and the arm acting as a regular pendulum. We again confirmed the effectiveness of the modified system through numerical simulations. The results indicate that the deceleration of the crib increases gradually and is maintained at around the target value of $25 \mathrm{G}$ when the deceleration of the base fixed on the vehicle seat is $30 \mathrm{G}$. The robustness of the proposed control system was also examined based on numerical simulations (Kawashima, 2015).

For this work, a semi-active acceleration control law was derived for controlling the acceleration of the crib directly, but is not required for the generation of the arm trajectory. The semi-active control system was built using a dynamic equation for the jerking of the crib. The effectiveness was confirmed using software for a multibody dynamics simulation. Some of the results are reported herein.

In our systems, arm 1, which is tilted backward and acts as an inverted pendulum, is supported by a stopper. The controlled joints are set for a large damping under normal conditions for a comfortable ride. Moreover, a forward stopper is also installed for safety. In addition, the controlled joints were designed to exhibit a large friction torque as a fail-safe in case of a breakdown of the control system.

For the impact control system, a method was proposed for calculating the seatbelt tension required to maintain an acceleration of the thorax, a deformation of the thorax, and the migration length of the occupant in the cabin within the tolerance limits during a car crash for a nonlinear human-vehicle system (Balandin et al., 2001). To add to the occupant safety of a modern vehicle, a crushable zone is designed for the vehicle body, and seatbelts and air bags are installed in the cabin. In addition, the use of a child-seat is obligatory when an infant is present. However, these are passive or openloop types systems, and do not always perform as expected owing to certain types of disturbances. Therefore, an impact control system that provides feedback regarding the condition of the crib is required to obtain a definitive result. In terms of active impact control, an optimal control system, an H infinity control system (Wang et al., 2005a), a system using feed-forward input (Wang et al., 2005b), and a gain-scheduled control system (Wang et al., 2007) have been investigated. In addition, an active knee bolster applying an impact control method for protecting the occupants from injury has been developed (Kato et al., 2007).

A semi-active impact control system, in which the actuator can be miniaturized and the power consumption can be reduced, has also been studied (Kawashima, 2005). The system utilizes an actuator for semi-active control using a braking mechanism. In addition, an active seatbelt that uses a semi-active actuator was proposed (Kawashima, 2008), and its effectiveness was confirmed experimentally using a model. A knee bolster applied for semi-active impact control has also been studied (Narukawa and Nishimura, 2012).

Although child restraint systems with moving mechanisms are not considered in the present technical standards, this paper demonstrates the extent to which the deceleration of a crib can be reduced when both a moving mechanism and a control system are applied.

This article is based on the paper presented at the $8^{\text {th }}$ Asian Conference on Multibody Dynamics hosted by Japan Society of Mechanical Engineers. 


\section{Nomenclature}

The symbols, representative values, and descriptions used in the present paper are as follows:

$A_{r}=-24.0 \mathrm{G}$ : Desired acceleration of the crib during vehicle movement, or the reference input.

$A_{r}=-21.6 \mathrm{G}$ : Desired acceleration of the crib after the vehicle stops, or the reference input.

$C_{2}=0.00 \mathrm{Nms} / \mathrm{rad}$ : Damping coefficient of the joint between arm 1, which acts as an inverted pendulum, and arm 2 , which acts as a regular pendulum.

$-D_{1}$ : Friction torque of the joint between the base and arm 1 . This is the control input.

$l_{1}=500 \mathrm{~mm}$ : Length of arm 1 .

$l_{2}=300 \mathrm{~mm}$ : Length of arm 2 .

$M=13.0 \mathrm{~kg}$ : Mass of the crib including the mass of the infant.

$m=1.00 \mathrm{~kg}$ : Mass of the joint between arm 1 and $\operatorname{arm} 2$.

$m_{1}=5.00 \mathrm{~kg}$ : Mass of arm 1 .

$m_{2}=3.00 \mathrm{~kg}$ : Mass of arm 2 .

$\ddot{X}=-30.0 \mathrm{G}$ : Acceleration of the vehicle, i.e., acceleration of the base. This is assumed to be a constant.

$\ddot{x}_{c}$ : Horizontal acceleration of the crib.

$\theta_{1}$ : Angular displacement of arm 1 .

$\theta_{2}$ : Absolute angular displacement of arm 2.

$\theta_{10}=-\pi / 6$ : Initial angular displacement of arm 1 .

$\theta_{12}=-\pi / 6$ : Difference between angular displacements of arms 1 and 2 , that is, $\theta_{12}=\theta_{1}-\theta_{2}$.

\section{Semi-active acceleration control law}

\subsection{Analytical model}

To propose the control law, an analytical model was derived, which is shown in Fig. 2(a). The crib and joint are assumed to be a single mass particle. In this study, for simplicity, arm 2 rotates at a difference of 30 degrees from arm 1 , that is, $\theta_{12}=\theta_{1}-\theta_{2}$ as shown in Fig. 2(b). In addition, the torque of the joint connecting arm 1 to the base, $D_{1}$, is only controlled using a brake mechanism.
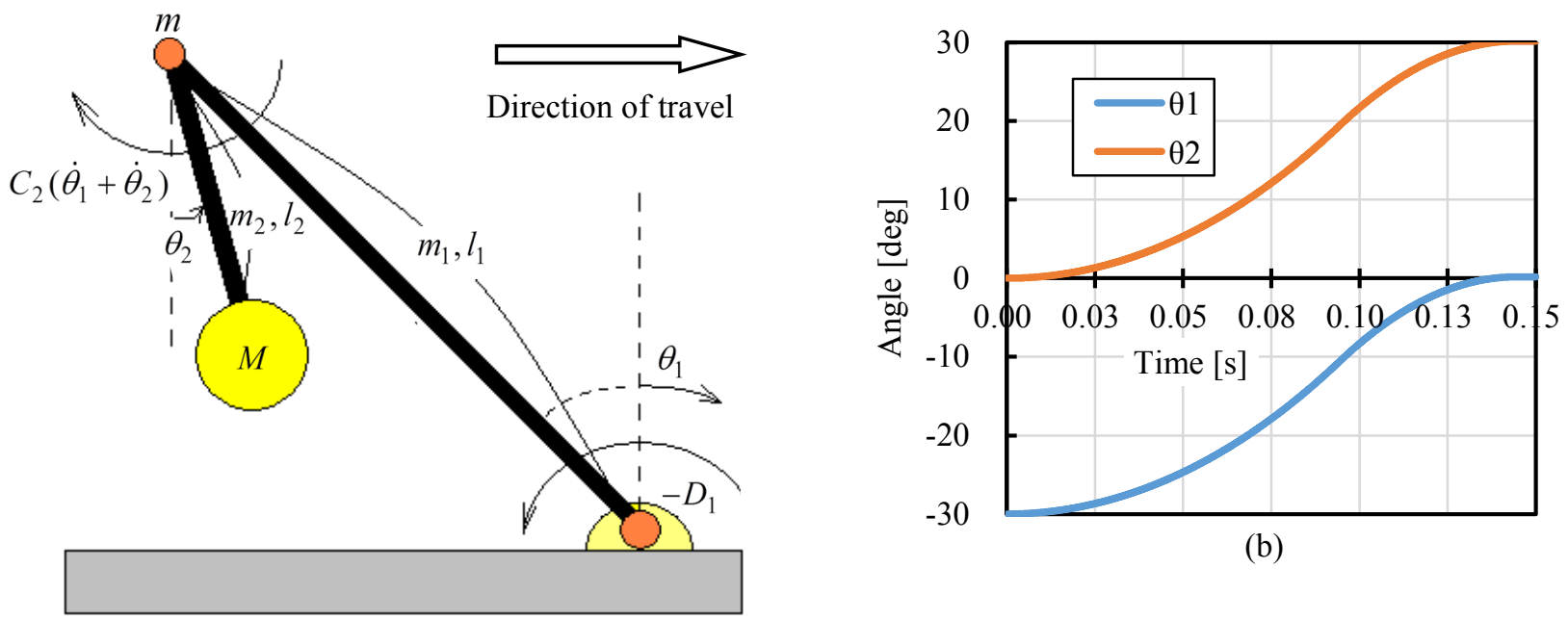

(b)

(a)

Fig. 2 Analytical model of the proposed in-car crib with joint application of regular and inverted pendulum mechanisms (a) and the relationship between $\theta_{1}$ and $\theta_{2}$ (b).

The equation of motion for the model shown in Fig. 2 can be derived as follows: 


$$
\begin{aligned}
{\left[\left(\frac{m_{1}}{3}+m+m_{2}\right.\right.} & \left.+M) l_{1}^{2}+\left(\frac{m_{2}}{3}+M\right) l_{2}^{2}+2\left(\frac{m_{2}}{2}+M\right) l_{1} l_{2} \cos \left(2 \theta_{1}-\theta_{12}\right)\right] \ddot{\theta}_{1} \\
& -2\left(\frac{m_{2}}{2}+M\right) l_{1} l_{2} \sin \left(2 \theta_{1}-\theta_{12}\right) \dot{\theta}_{1}{ }^{2}+4 C_{2} \dot{\theta}_{1}-\left(\frac{m_{1}}{2}+m+m_{2}+M\right) l_{1} g \sin \left(\theta_{1}\right) \\
& +\left(\frac{m_{2}}{2}+M\right) l_{2} g \sin \left(\theta_{1}-\theta_{12}\right) \\
& =D_{1}-\left[\left(\frac{m_{1}}{2}+m+m_{2}+M\right) l_{1} \cos \left(\theta_{1}\right)+\left(\frac{m_{2}}{2}+M\right) l_{2} \cos \left(\theta_{1}-\theta_{12}\right)\right] \ddot{X}
\end{aligned}
$$

\subsection{Proportional control law}

First, for a comparison with the sliding mode control law, a semi-active proportional control law using feedback regarding the horizontal acceleration of the crib is proposed as follows:

$$
u=-K_{p}\left(\ddot{x}_{c}-A_{r}\right), \text { if } \dot{\theta}_{1} \cdot u<0 \text {, then } D_{1}=u \text {, else } D_{1}=0 .
$$

Here, $K_{p}$ is the proportional gain of the feedback control.

\subsection{Sliding mode control law}

Next, a semi-active sliding mode control law is proposed because the system is nonlinear. The Eq. of motion (1) is differentiated to derive the acceleration control law as follows:

$$
\begin{aligned}
{\left[\left(\frac{m_{1}}{3}+m+m_{2}\right.\right.} & \left.+M) l_{1}^{2}+\left(\frac{m_{2}}{3}+M\right) l_{2}^{2}+2\left(\frac{m_{2}}{2}+M\right) l_{1} l_{2} \cos \left(2 \theta_{1}-\theta_{12}\right)\right] \dddot{\theta}_{1} \\
& -4\left(\frac{m_{2}}{2}+M\right) l_{1} l_{2}\left[2 \sin \left(2 \theta_{1}-\theta_{12}\right) \ddot{\theta}_{1}+\cos \left(2 \theta_{1}-\theta_{12}\right) \dot{\theta}_{1}^{2}\right] \dot{\theta}_{1}+4 C_{2} \ddot{\theta}_{1} \\
& -\left[\left(\frac{m_{1}}{2}+m+m_{2}+M\right) l_{1} \cos \left(\theta_{1}\right)-\left(\frac{m_{2}}{2}+M\right) l_{2} \cos \left(\theta_{1}-\theta_{12}\right)\right] g \dot{\theta}_{1} \\
& =\dot{D}_{1}-\left[\left(\frac{m_{1}}{2}+m+m_{2}+M\right) l_{1} \cos \left(\theta_{1}\right)+\left(\frac{m_{2}}{2}+M\right) l_{2} \cos \left(\theta_{1}-\theta_{12}\right)\right] \dddot{X} \\
& +\left[\left(\frac{m_{1}}{2}+m+m_{2}+M\right) l_{1} \sin \left(\theta_{1}\right)+\left(\frac{m_{2}}{2}+M\right) l_{2} \sin \left(\theta_{1}-\theta_{12}\right)\right] \ddot{X} \dot{\theta}_{1}
\end{aligned}
$$

The state equation is then arranged from this equation under the assumption that the vehicle deceleration is constant as follows:

$$
\{\dot{\theta}\}=\{f\}+\{B\} \dot{D}_{1}
$$

Here, $\{\theta\}=\left\{\begin{array}{lll}\theta_{1} & \dot{\theta}_{1} & \ddot{\theta}_{1}\end{array}\right\}^{\mathrm{T}}$ is the state variable vector, $f_{1}=\dot{\theta}_{1}, f_{2}=\ddot{\theta}_{1}$,

$$
\begin{aligned}
& f_{3}=\left\{\left[\left(m_{1} / 2+m+m_{2}+M\right) l_{1} \sin \left(\theta_{1}\right)+\left(m_{2} / 2+M\right) l_{2} \sin \left(\theta_{1}-\theta_{12}\right)\right] \ddot{X} \dot{\theta}_{1}-4 C_{2} \ddot{\theta}_{1}\right. \\
& +4\left(m_{2} / 2+M\right) l_{1} l_{2}\left[2 \sin \left(2 \theta_{1}-\theta_{12}\right) \ddot{\theta}_{1}+\cos \left(2 \theta_{1}-\theta_{12}\right) \dot{\theta}_{1}^{2}\right] \dot{\theta}_{1} \\
& \left.+\left[\left(m_{1} / 2+m+m_{2}+M\right) l_{1} \cos \left(\theta_{1}\right)-\left(m_{2} / 2+M\right) l_{2} \cos \left(\theta_{1}-\theta_{12}\right)\right] g \dot{\theta}_{1}\right\} / \bar{M}, \\
& B_{1}=B_{2}=0, B_{3}=1 / \bar{M} \text {, and } \\
& \bar{M}=\left(m_{1} / 3+m+m_{2}+M\right) l_{1}^{2}+\left(m_{2} / 3+M\right) l_{2}^{2}+2\left(m_{2} / 2+M\right) l_{1} l_{2} \cos \left(2 \theta_{1}-\theta_{12}\right) \text {. }
\end{aligned}
$$

The hyper-plane for the sliding mode control is selected to control the acceleration of the crib directly as follows:

$$
s=\ddot{x}_{c}-A_{r} .
$$

The semi-active sliding mode control law is obtained as follows (Hashimoto, 1993): 


$$
\dot{u}=-\alpha F(\{\theta\} \quad t) \frac{s^{*}}{\left|s^{*}\right|+\delta}, u=\int \dot{u} d t, \text { if } \dot{\theta}_{1} \cdot u<0 \text {, then } D_{1}=u \text {, else } D_{1}=0 \text {. }
$$

Here, a smooth function is used instead of a sign function (the signum function) to suppress the chatter of the control input. Additionally, $1<\alpha, 0<\left|u_{e q}\right|<F(\{\theta\} t), u_{e q}=-(\{G\}\{B\})^{-1}\{G\}\{f\}, \quad\{G\}=\partial s / \partial\{\theta\}$, $\operatorname{det}(\{G\}\{B\}) \neq 0, s^{*}=(\{G\}\{B\})^{\mathrm{T}} s$, and $0<\delta$.

\section{Simulation and results}

\subsection{Simulation model}

The effectiveness of the proposed control law was confirmed through simulations using software for a multibody dynamics simulation (Adams, MSC Software Co.). A simulation model of an in-car crib with the joint application of regular and inverted pendulum mechanisms is shown in Fig. 3. The arms and crib are modeled as a uniform solid body.

For the seat arrangement, the cabin of a minivan was applied. The size of the crib is $300 \mathrm{~mm} \times 900 \mathrm{~mm} \times 310 \mathrm{~mm}$, which is set based on the 97th percentile of Japanese 18 month old Japanese boys (Takaishi, 1991). Arm 2, acting as a regular pendulum, is linked with arm 1, acting as an inverted pendulum, and arm 2 rotates at a difference of 30.0 degrees from arm 1. The brake mechanism at the joint connecting the base and arm 1 was simulated using a motor element. The torque was obtained from Eq. (2) or Eq. (6). The deceleration of the vehicle was assumed to be constant at $30.0 \mathrm{G}$ through a deformation of the crushable zone, and the initial velocity of the car crash was set at $100 \mathrm{~km} / \mathrm{h}$, that is, the vehicle decelerated for $0.0944 \mathrm{~s}$. The desired horizontal acceleration of the crib controller was set to $-24.0 \mathrm{G}$ during vehicle movement, and to $-21.6 \mathrm{G}$ after the vehicle stopped. And the time step of the simulation with the feedback control was set to $0.1 \mathrm{~ms}$.

The control objective of the present study was for the horizontal acceleration of the crib to decrease within a limited space, that is, the horizontal acceleration of the crib was to be kept at the constant desired value, which is dependent on the movement distance of the crib. Therefore, the desired value should be set in consideration of the cabin space. In this study, it was decided as the maximum deceleration without the crib bumping into the front seat back in the minivan cabin.

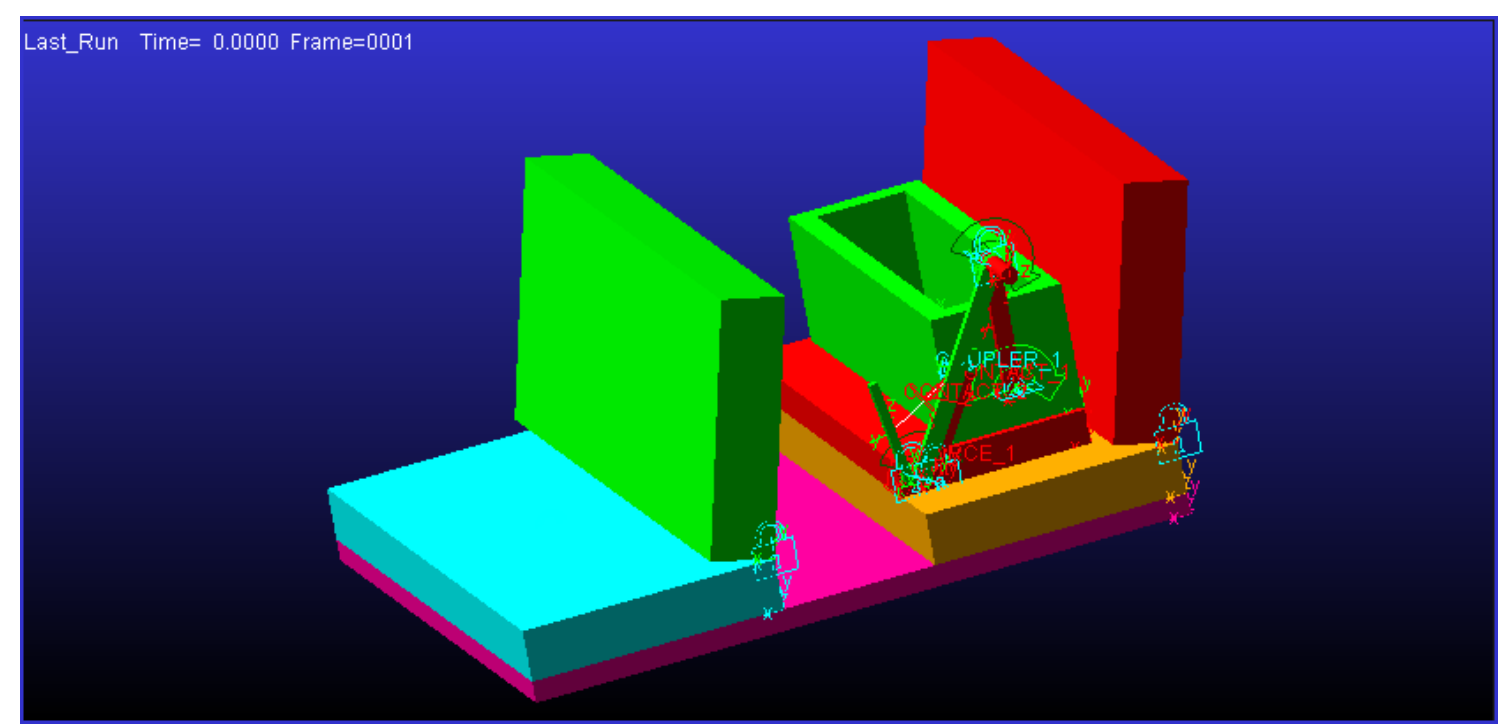

Fig. 3 Simulation model of the proposed in-car crib with joint application of regular and inverted pendulum mechanisms.

\subsection{Simulation results without control}

Figure 4 shows the simulation results for a case in which the friction torque of the joint connecting the base and arm 1 is constant, that is, without control. The joint torque $D_{1}$ was set at $-2.50 \times 10^{6} \mathrm{Nm}$, which was determined through trial-and-error, and thus the rotation of arm 1, acting as an inverted pendulum, reaches about 30 degrees, that is, arm 1 
rotates from -30 degrees to around zero degrees in angular displacement.

The blue, red, gray, and orange solid lines indicate the changes in the vehicle acceleration, $\ddot{X}$; the horizontal acceleration of the crib, $\ddot{x}_{c}$; the angular displacement of arm $1, \theta_{1}$; and the joint torque, $D_{1}$, respectively.

In the case of no control, the initial horizontal deceleration of the crib is larger than the desired value of $24.0 \mathrm{G}$, which then decreases to less than the desired value while the vehicle decelerates, and becomes smaller than the desired value after the vehicle stops, although the rotation of the arm 1 is about 30 degrees. Therefore, to maintain the horizontal deceleration of the crib below the desired value, a longer crib movement is required.

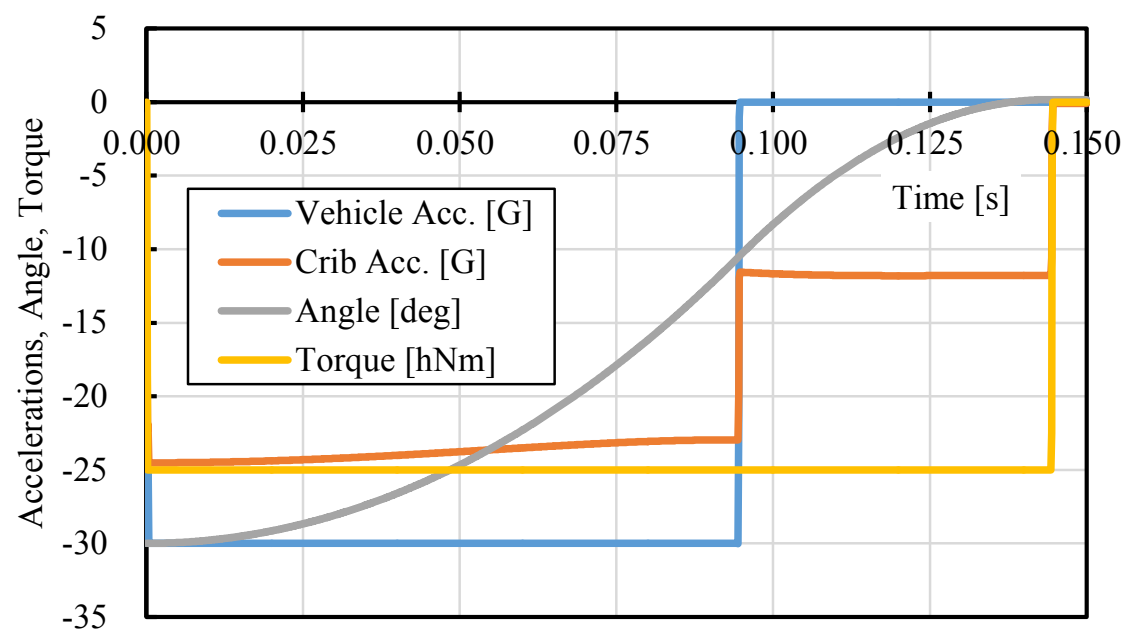

Fig. 4 Simulation results for no control when the friction torque of the joint connecting the base and arm 1, acting as an inverted pendulum, is constant at $D_{1}=-2.50 \times 10^{6} \mathrm{Nm}$. Although the rotation of arm 1 is about 30 degrees, the initial horizontal deceleration of the crib is larger than the desired value of $24.0 \mathrm{G}$, and then decreases to less than the desired value while the vehicle decelerates, becoming smaller than the desired value after the vehicle stops.

\subsection{Simulation results with proportional control}

Figure 5 shows the simulation results (a) and the error of the crib deceleration from the desired value (b) for when proportional control is applied. The proportional gain $K_{p}$ is set at $2000 \mathrm{~kg} \cdot \mathrm{m}$. The desired deceleration of the crib after the vehicle stops is set at $90 \%$ of the desired value while the vehicle decelerates for margin of the error caused by rapid change of the vehicle deceleration.

As a result, the joint torque varies, and the horizontal deceleration of the crib can be maintained at nearly the desired value, although it does vary slightly and the steady-state error is remained. The effectiveness of the control system is therefore illustrated. The steady-state error increases according to the decrease in proportional gain. The movement distance of the crib is small because the crib deceleration is controlled immediately from the start.

\subsection{Simulation results with proportional control and disturbance}

To confirm the robustness of the proportional controller, a sinusoidal disturbance with an amplitude of $1.50 \mathrm{G}$ and a frequency of $30.0 \mathrm{~Hz}$ is added to the vehicle deceleration for $0.100 \mathrm{~s}$. Figure 6 shows the simulation results (a) and the error (b) when proportional control and the disturbance are both applied.

As the results indicate, the horizontal deceleration of the crib is maintained near the desired value, although it does slightly vary with the fluctuations in the vehicle deceleration and the steady-state error is remained. 

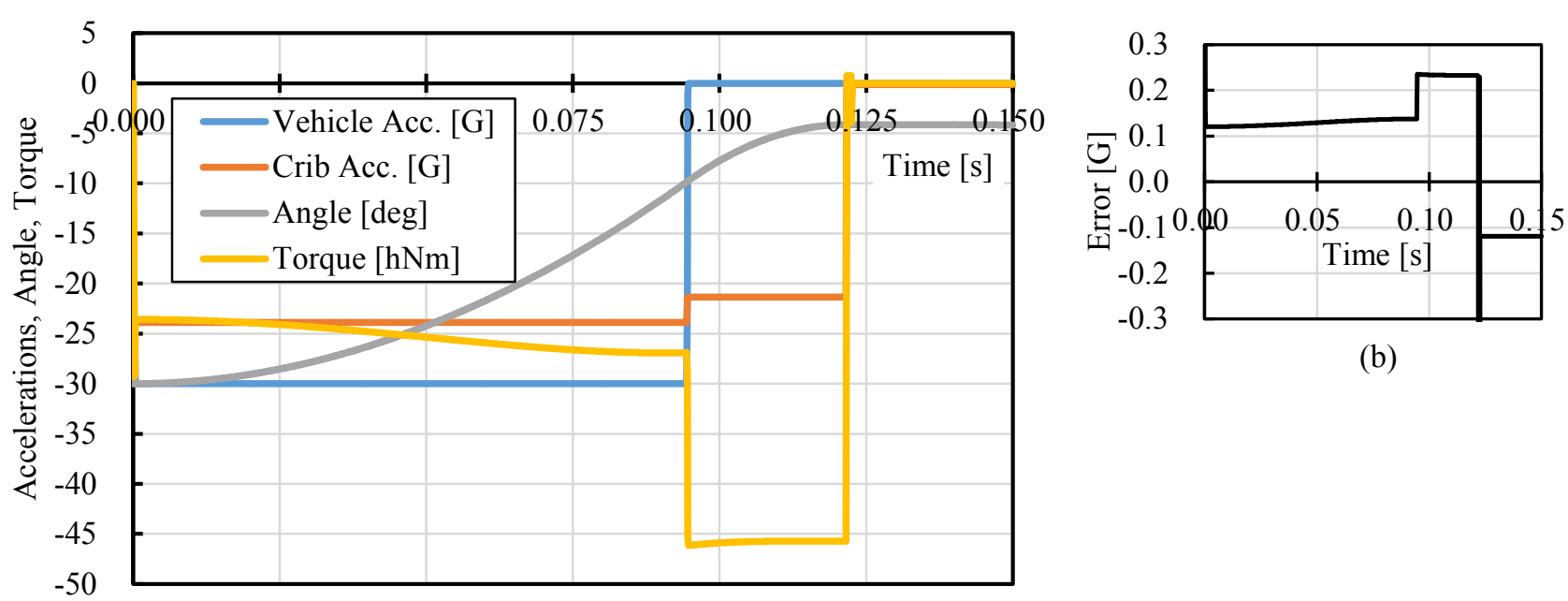

(b)

(a)

Fig. 5 Simulation results (a) and the error of the crib deceleration from the desired value (b) for when proportional control is applied with a proportional gain of $K_{p}=2000 \mathrm{~kg} \cdot \mathrm{m}$. The joint torque varies and the horizontal deceleration of the crib can be maintained at around the desired value, although it does vary slightly and the steady-state error is remained. The effectiveness of the control system is therefore illustrated.
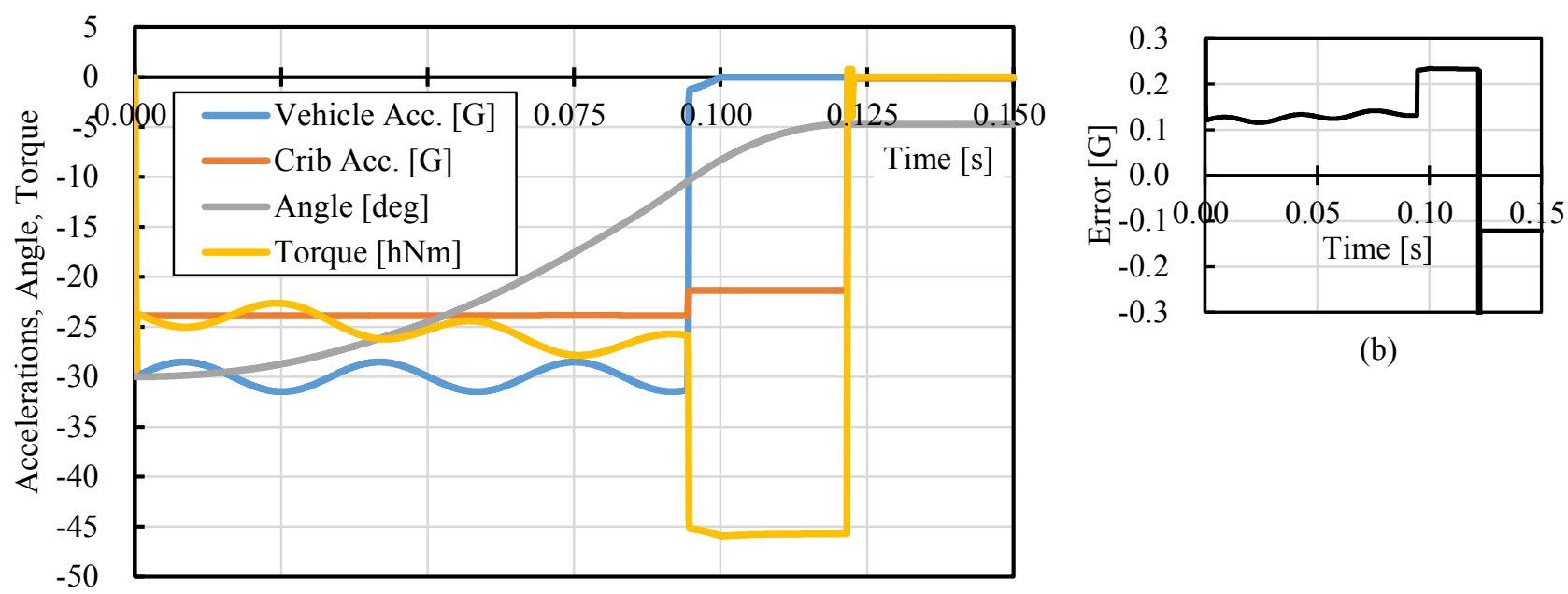

(b)

Fig. 6 Simulation results (a) and the error of the crib deceleration from the desired value (b) for when proportional control, with a proportional gain of $K_{p}=2000 \mathrm{~kg} \cdot \mathrm{m}$, and a sinusoidal disturbance with an amplitude of $1.50 \mathrm{G}$ and a frequency of $30.0 \mathrm{~Hz}$ added to the vehicle deceleration for $0.100 \mathrm{~s}$ are both applied. The horizontal deceleration of the crib is maintained at near the desired value, although it varies slightly with the fluctuations in the vehicle deceleration and the steady-state error is remained.

\subsection{Simulation results with sliding mode control}

Figure 7 shows the simulation results (a) and the error of the crib acceleration from the desired value (b) with sliding mode control. The parameters of the control law in Eq. (6) are set at $\alpha=25.0$ and $\delta=0.0100$. The initial value of differential Eq. (6) is set at $-1.25 \times 10^{6} \mathrm{Nm}$. These parameters were determined through trial-and-error.

As a result, the joint torque varies, and the horizontal deceleration of the crib gradually increases to the desired value where it remains constant without steady-state error. It is therefore illustrated that the sliding mode control system is effective, and that the crib movement is the shortest when the crib deceleration is maintained at the desired value. The 
horizontal acceleration of the crib converges to the desired value more quickly and becomes unstable as parameter $\alpha$ increases. The initial deceleration of the crib depends on the initial value of the differential equation.
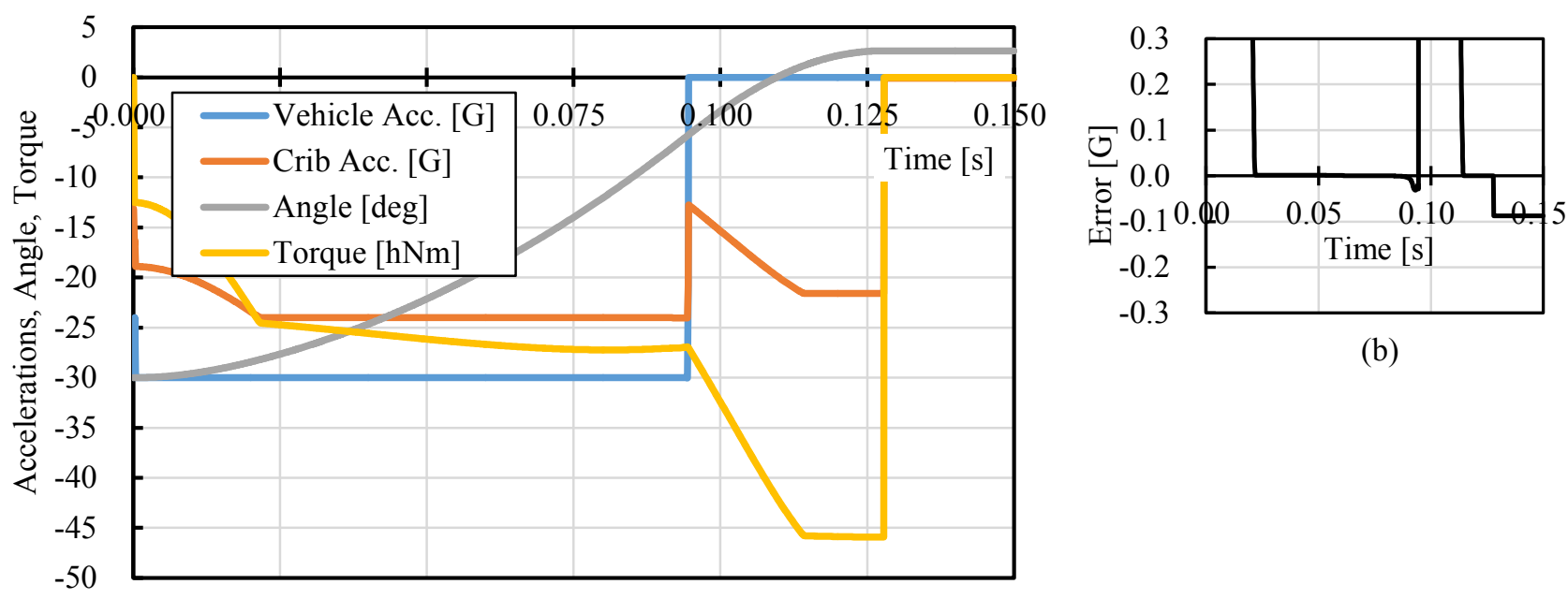

(b)

(a)

Fig. 7 Simulation results (a) and the error of the crib deceleration from the desired value (b) when sliding mode control is applied, the parameters of which are set at $\alpha=25.0$ and $\delta=0.0100$, and the initial value of differential Eq. (6) is set at $-1.25 \times 10^{6} \mathrm{Nm}$. The joint torque varies and the horizontal deceleration of the crib guradually increases to the desired value, where it remains constant without steady-state error. It is therefore illustrated that the sliding mode control system is effective, and that the crib movement is the shortest when the crib deceleration is maintained at the desired value.

\subsection{Simulation results with sliding mode control and disturbance}

To confirm the robustness of the proposed controller, a sinusoidal disturbance with an amplitude of $1.50 \mathrm{G}$ and a frequency of $30.0 \mathrm{~Hz}$ is added to the vehicle deceleration for $0.100 \mathrm{~s}$. Figure 8 shows the simulation results (a) and the error (b) when sliding mode control and the disturbance are both applied.
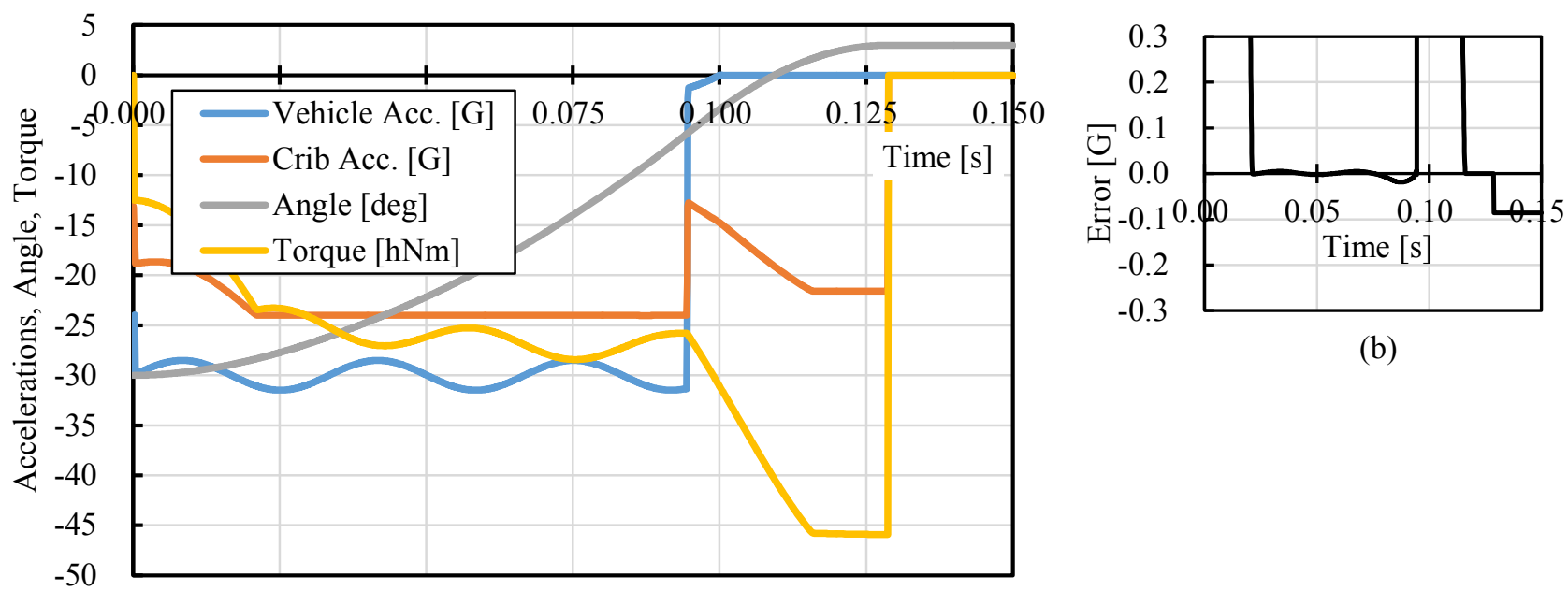

(b)

(a)

Fig. 8 Simulation results (a) and the error of the crib deceleration from the desired value (b) when applying sliding mode control, with parameters of $\alpha=25.0$ and $\delta=0.0100$, and a sinusoidal disturbance with an amplitude of $1.50 \mathrm{G}$ and a frequency of $30.0 \mathrm{~Hz}$ added to the vehicle deceleration for $0.100 \mathrm{~s}$. The initial value of differential Eq. (6) is set at $-1.25 \times 10^{6} \mathrm{Nm}$. The joint torque varies, and the horizontal deceleration of the crib gradually increases to the desired value, where it remains constant without steady-state error despite the fluctuations in the vehicle deceleration. The robustness of the proposed control system for the vehicle deceleration is therefore confirmed. 
As the results indicate, the joint torque varies, and the horizontal deceleration of the crib increases to the desired value where it remains constant without steady-state error despite the fluctuations in the vehicle deceleration. The robustness of the proposed control system for the vehicle deceleration are therefore confirmed. However, it is necessary to pay the attention to the integration performance of the controller.

\section{Conclusions}

A semi-active acceleration control system of an in-car crib with a joint application of regular and inverted pendulum mechanisms was proposed for direct control of the horizontal acceleration of the crib. In addition, the effectiveness of the control system was confirmed using software for a multibody dynamics simulation. As the results indicate, the following aspects were clarified:

(1) The proposed acceleration control system was found to be effective when comparing a crib system with proportional control to a system without such control.

(2) The sliding mode control system derived using a dynamic equation for the jerking of the crib system was also found to be effective.

(3) The sliding mode control system was determined to be robust under vehicle deceleration.

\section{Acknowledgment}

This work was supported by a Grant-in-Aid for Scientific Research (C) (JSPS KAKENHI Grant Number: JP24560279).

\section{References}

Balandin, D. V., Bolotnik, N. N. and Pilkey, W. D., Optimal Protection From Impact, Shock, and Vibration (2001), pp.315-325, Gordon and Breach Science Publishers.

Hashimoto, H., Working(!?) Theory of Nonlinear Control III: Sliding Mode Control (exposition), SYSTEMS, CONTROL and INFORMATION (in Japanese), Vol.37, No.6 (1993), pp.371-378.

Kato, M., Nishimura, H., Amano, Y. and Shimogo, T., Injury protection control of occupant legs by active knee bolster, Transactions of the Japan Society of Mechanical Engineers (in Japanese), Series C, Vol.73, No.736 (2007), pp.31853192.

Kawashima, T., Study for active controlled pendulum type bed for vehicle (Prevention of the patient sliding on the bed), Transactions of the Japan Society of Mechanical Engineers (in Japanese), Series C, Vol.68, No.670 (2002), pp.16261632.

Kawashima, T., Research on semi-active shock control (Transmission of necessary minimum impulsive force), Transactions of the Japan Society of Mechanical Engineers (in Japanese), Series C, Vol.71, No.705 (2005), pp.15211528.

Kawashima, T., Research on active seat belt, Transactions of the Japan Society of Mechanical Engineers (in Japanese), Series C, Vol.74, No.739 (2008), pp.587-594.

Kawashima, T., Basic research on inverted pendulum style child bed, Proceedings of the 5th Asian Conference on Multibody Dynamics (2010), Kyoto, Japan, No.59750.

Kawashima, T., Research on semiactive child car bed with joint application of regular and inverted pendulum mechanisms, Proceedings of the 6th Asian Conference on Multibody Dynamics (2012), Shanghai, China, ID137000.

Kawashima, T., Basic research on semi-active in-car crib with joint application of regular and inverted pendulum mechanisms (Study on the mechanism and the control system), Transactions of the Japan Society of Mechanical Engineers (in Japanese), Vol.80, No.812 (2014), DOI:10.1299/transjsme.2014trans000X.

Kawashima, T., Control system for semi-active in-car crib with joint application of regular and inverted pendulum mechanisms, Bulletin of the JSME, Mechanical Engineering Journal, Vol.2, No.3 (2015), DIO:10.1299/mej.1400557.

Narukawa, T. and Nishimura, H., Control system design for occupant lower extremity protection using semi-active knee bolster, Transactions of the Japan Society of Mechanical Engineers (in Japanese), Series C, Vol.78, No.789 (2012), 
pp.1711-1722.

Ono, M., Mimura, E., Otsuka, T. and Ishimoto, S., Child seat device for vehicle, Japanese patent 4211182 (2008).

Sawaishi, T., The rotary type and impact absorption type child-seat and the rolling mechanism for the child-seat, Japanese patent disclosure P2004-299660A (2004).

Takaishi, M., Kato, N., Ohmori, S. and Ohe, H., On the result of growth monitoring about babies and little children in 1990. The Journal of Child Health (in Japanese), Vol. 50, No. 6 (1991), pp.671-680.

Tamura, K., Rotating safety child seat, Japanese utility model registration 3068346 (2000).

Wang, D., Nishimura, H. and Shimogo, T., Active control of shock: Applications of LQI control and Hळ control, Transactions of the Japan Society of Mechanical Engineers (in Japanese), Series C, Vol.71, No.704 (2005a), pp.1223-1230.

Wang, D., Nishimura, H., Shimogo, T. and Amano, Y., Active control of shock by using feedforward input, Transactions of the Japan Society of Mechanical Engineers (in Japanese), Series C, Vol.71, No.710 (2005b), pp.2912-2919.

Wang, D., Nishimura, H. and Shimogo, T., Active control of shock by gain scheduling, Journal of Sound and Vibration, Vol.308 (2007), pp.647-659. 